\title{
Terceirização e impactos para a saúde e trabalho: Uma revisão sistemática da literatura
}

Outsourcing and impacts for health and work: A systematic review of the literature Subcontratación e impactos en la salud e en el trabajo: Una revisión bibliográfica sistemática

\author{
Marina Bernardo MANDARINI ${ }^{1}$ \\ Amanda Martins ALVES \\ Marina Greghi STICCA \\ Universidade de São Paulo, Faculdade de Filosofia, Ciências e Letras de Ribeirão Preto, Ribeirão Preto, SP, Brasil
}

ResumO A terceirização acarreta diversas modificações nas atividades e contextos de trabalho, e impacta, também, a saúde do trabalhador. No entanto, a revisão da literatura aponta que as pesquisas realizadas na área focam, majoritariamente, aspectos financeiros e administrativos deste processo. Esta revisão sistemática buscou discorrer sobre a produção científica em relação à terceirização e seus impactos para a saúde, família e trabalho dos profissionais terceirizados. As bases de dados consultadas foram SciELO, ProQuest, PsycINFO e Scopus, no período de 2000 a 2015. Foram encontrados 1.754 artigos na fase de seleção inicial, sendo que 20 satisfizeram os critérios de inclusão. Os resultados evidenciaram impactos negativos na saúde dos trabalhadores terceirizados. Foram identificados nos estudos fatores desencadeadores de estresse, doenças relacionadas ao trabalho, riscos de acidentes, falta de suporte à saúde e à segurança, adoecimento psíquico, insegurança e esvaziamento do sentido atribuído ao trabalho. Também foram identificados artigos em que a precarização das condições de trabalho foi associada à terceirização, caracterizada por diferenças salariais e de benefícios, perda dos direitos trabalhistas, excesso de trabalho e falta de treinamento. A partir da revisão realizada, verificou-se escassez de estudos que focassem os impactos do processo de terceirização para a saúde dos profissionais terceirizados, evidenciando a necessidade de aprofundar as discussões sobre o tema.

Palavras-chave:

Terceirização; revisão; saúde.

\section{Abstract}

Outsourcing entails various changes in work activities and contexts, and also has impacts on worker health. However, the literature shows that the research conducted in this area focuses mainly on financial and administrative aspects of this process. This systematic review aimed to discuss the scientific production in relation to outsourcing and its impact on the health, family, and work of outsourced professionals. The databases consulted were SciELO, ProQuest, PsycINFO, and Scopus, for the period from 2000 to 2015. In the initial selection phase, 1,754 articles were found, of which 20 met the inclusion criteria. The results showed negative impacts on the health of contract workers. In these studies we identified trigger factors of stress, work-related diseases, risk of accidents, lack of support for health and safety, mental illness, insecurity, and emptiness of meaning attributed to work. Articles were also identified in which precarious working conditions were associated with outsourcing, characterized by differences in salaries and benefits, loss of labor rights, overwork, and lack of training. Based on the review conducted, there was a scarcity of studies that focused on the impacts of the outsourcing process on the health of outsourced professionals, highlighting the need for further discussions on the topic.

Keywords:

Outsourcing; review; health.

Endereço para correspondência: Faculdade de Filosofia, Ciências e Letras de Ribeirão Preto, Universidade de São Paulo, Departamento de Psicologia, Av. Bandeirantes, 3900, Monte Alegre, Ribeirão Preto, Brasil 14040-901. Telefone: (16) $3315-9091$. Email: marinamandarini@gmail.com 
Resumen La subcontratación implica diversos cambios en las actividades y contextos del trabajo, y causa impacto también en la salud del trabajador. Sin embargo, tras una revisión bibliográfica, se ha percatado que las investigaciones llevadas a cabo en el área se centran principalmente en los aspectos financieros y administrativos de ese proceso. Esta revisión sistemática ha buscado discutir la producción científica en relación con la subcontratación y sus impactos en la salud, en la familia y en el trabajo de los profesionales subcontratados. Las bases de datos que se consultaron fueron SciELO, ProQuest, PsycINFO y Scopus, en el período 2000-2015. Se han encontrado, en la fase de selección inicial, con 1.754 artículos, entre los cuales 20 cumplieron los criterios de inclusión. Los resultados mostraron impactos negativos en la salud de los trabajadores subcontratados. Se han identificado en estudios factores desencadenantes de estrés, enfermedades relacionadas con el trabajo, riesgo de accidentes, falta de apoyo a la salud y a la seguridad, enfermedad mental, inseguridad y vaciedad del significado atribuido al trabajo. Además, se han identificado artículos en los que la precarización de las condiciones de trabajo fue asociada a la subcontratación, que se caracteriza por las diferencias salariales y de beneficios, la pérdida de los derechos laborales, el exceso de trabajo y la falta de práctica. A partir de la revisión realizada, se ha verificado escasez de estudios que focalizasen los impactos del proceso de subcontratación en la salud de los profesionales, lo que evidenció la necesidad de profundizarse discusiones sobre el tema.

Palabras-clave:

Subcontratación; revisión; salud.

A partir da década de 1990, com a internacionalização de mercados, o aumento da competitividade e o avanço tecnológico, tem-se verificado novos desenhos organizacionais, flexibilização e mudanças nas relações de trabalho instituídas dentro das novas práticas de gestão. Essas mudanças tiveram início no fim dos anos 1960 e início dos anos 1970 com a crise do sistema taylorista-fordista, sendo necessária uma transição para um novo formato de acumulação, que ficou conhecido como flexível, e que acarretou mudanças nos processos de trabalho e no contexto organizacional. A nova estrutura produtiva das empresas passou a ser flexível, o que possibilitou a elas recorrer à diminuição de seu tamanho (downsizing), à terceirização de atividades (outsourcing) e à adoção de novas formas de organização dos processos internos.

A implementação desse novo modelo resultou em novas formas flexíveis de trabalho e, consequentemente, na reconfiguração das relações entre capital e trabalho e em novas formas de organizar a produção e os próprios trabalhadores (Lima, 2010). Tais formas flexíveis constituem uma alternativa para que as organizações se adaptem às modificações no cenário mundial, como a necessidade de atingir altos níveis de competitividade, a imposição da redução de custos em consequência da maior liberalização comercial e adequação às mudanças tecnológicas. As práticas flexíveis envolvem a ampliação das responsabilidades dos trabalhadores e do seu grau de envolvimento e formas alternativas de contratação, como a terceirização, o contrato temporário ou eventual, o trabalho em tempo parcial, o salário e as jornadas flexíveis. Também são considerados nessa categoria o enriquecimento de cargos e a rotação, e o aumento das funções exercidas por um mesmo trabalhador.

O panorama mundial indica a adoção de práticas flexíveis por vários países na Europa e uma grande utilização nos Estados Unidos. No Brasil, levantamentos sobre o grau de utilização de empregos flexíveis por empresas do país apontam a terceirização como a modalidade especial de contrato de trabalho mais utilizada. Tal prática consiste na subcontratação de empresas que oferecem o serviço de trabalhadores para executar e desenvolver atividades secundárias ou periféricas do processo produtivo. O processo de terceirização pode adquirir diferentes formas, como operações pontuais, contratos de longo prazo e alianças estratégicas envolvendo contratos que garantem exclusividade entre as partes, entre outras.

De modo geral, as alterações decorrentes da reorganização da produção e dos novos arranjos organizacionais que caracterizam a terceirização modificam a natureza das tarefas, a distribuição das atividades e a organização dos relacionamentos entre as empresas, interferindo também na interação entre os indivíduos de ambas as partes (Davis-Blake \& Broschak, 2009).

Apesar da existência de algumas produções científicas que abordam os impactos da terceirização para os trabalhadores, a literatura aponta que os estudos relacionados à terceirização têm como foco principal identificar as razões quanto à decisão de terceirizar certo serviço ou não, o objetivo das empresas ao utilizar a terceirização, o gerenciamento da relação de terceirização e as razões implicadas no sucesso ou falha da implantação da terceirização (Brooks, 2006). Isso demonstra que o foco das pesquisas nessa área é essencialmente voltado para aspectos associados aos contextos financeiro e administrativo. 
Como há indicações de lacunas de pesquisa e escassez de artigos em relação ao tema, a presente revisão de literatura buscou discorrer sobre a produção científica em relação à terceirização, tema que está sendo discutido no cenário brasileiro por conta de propostas de alterações trabalhistas, e sobre os impactos para a saúde, a família e o trabalho dos profissionais que aderem a tal prática.

\section{MÉTODO}

Segundo Petticrew e Roberts (2006), a revisão sistemática é um método que possibilita organizar informações e contribuir para responder a questões de pesquisa, bem como mapear áreas com poucos estudos relevantes e identificar a necessidade de novas pesquisas. É, portanto, um método de pesquisa de caráter descritivo e exploratório. A revisão sistemática utilizada neste estudo foi baseada no guia prático para revisões sistemáticas no campo das ciências sociais, publicado por Petticrew e Roberts (2006), e nas sugestões para produção de um artigo de revisão sistemática, elaboradas por Zoltowski, Costa, Teixeira e Koller (2014).

A revisão sistemática da literatura foi realizada a partir da análise de artigos científicos nacionais e internacionais disponíveis nas bases eletrônicas de dados SciELO, ProQuest, PsycINFO e Scopus. Tais bases foram escolhidas por serem referência nas áreas de psicologia e administração, disciplinas que abordam o tema. Foram utilizadas as palavras-chave terceirização (outsourcing), saúde (health), trabalho (work) e/ou família (family). As palavras-chave foram selecionadas após pesquisa de descritores nas bases utilizadas e no Vocabulário de Termos em Psicologia, cadastrados na Biblioteca Virtual em Saúde Psicologia (BVS-Psi). A pesquisa bibliográfica foi realizada no período compreendido entre junho e julho de 2015, considerando trabalhos publicados de 2000 a 2015.

Para a organização e sistematização dos trabalhos encontrados, foi utilizado o software State of Art through Systematic Review (START), versão 1.6.3, desenvolvido pelo Laboratório de pesquisa em Engenharia de Software da Universidade Federal de São Carlos (UFSCar). Essa ferramenta auxilia o planejamento, a execução e a análise final dos estudos encontrados, tornando a revisão sistemática mais ágil, precisa e replicável (Hernandes, Zamboni, Fabbri, \& Thommazo, 2012). Assim como a busca pelos artigos, a avaliação dos mesmos foi realizada por dois pesquisadores da área, de acordo com os seguintes critérios de inclusão: (a) trabalhos publicados na língua inglesa e portuguesa, (b) artigos científicos e (c) estudos com a temática da terceirização e impactos para os trabalhadores terceirizados. Os critérios de exclusão foram: (a) livros, capítulos de livros, dissertações e teses; e (b) trabalhos que, apesar de apresentarem a terceirização e seus efeitos, não abordavam impactos para os trabalhadores terceirizados.

A seleção inicial resultou em 1.754 artigos, conforme indicado na Tabela 1. Os resumos desses estudos foram analisados, sendo excluídos os artigos que não abordavam o tema da terceirização e os impactos para os trabalhadores, e também os artigos duplicados, resultando em um total de 62 artigos identificados como relevantes e 1.692 artigos descartados. A partir dessa seleção inicial, os artigos considerados relevantes foram lidos e analisados por dois pesquisadores, sendo selecionados apenas os estudos que tratavam dos impactos da terceirização para os trabalhadores terceirizados, resultando em 20 artigos selecionados (Tabela 1). Ao longo de todo o processo de seleção e análise dos dados, os pesquisadores verificaram os critérios de inclusão e exclusão e discutiram sobre os estudos, a fim de alinhar a forma de análise dos dados.

As informações dos artigos selecionados foram sintetizadas em uma tabela, contemplando os seguintes itens: metodologia, procedimentos e tipos de pesquisa (Tabela 2). Os estudos também foram organizados e analisados em duas categorias temáticas predominantes (Tabela 3). As categorias foram elaboradas e validadas por três pesquisadores da área, que, a partir da leitura dos artigos, identificaram as ideias centrais abordadas, e sugeriram em reuniões de consenso duas categorias temáticas predominantes: (a) Impactos na saúde dos trabalhadores; e (b) Impactos nas condições de trabalho. Tais categorias foram divididas conforme os subtemas identificados nos artigos selecionados.

\section{RESULTADOS}

A Tabela 1 mostra os resultados, em termos quantitativos, das buscas realizadas nas bases de dados citadas. Em síntese, foram encontrados 1.754 estudos indexados nas quatro bases de dados; destes, 681 eram artigos duplicados e 62 foram considerados relevantes. Foram lidos os resumos das 62 publicações consideradas relevantes, das quais 20 foram selecionadas por satisfazer os critérios de inclusão. 
TABELA 1. Número de artigos encontrados em cada base de dados e número de artigos selecionados

\begin{tabular}{ccc} 
& \multicolumn{2}{c}{ Base de dados } \\
\cline { 2 - 3 } & Levantados & Selecionados \\
ProQuest & 643 & 3 \\
PsycINFO & 928 & 4 \\
SciELO & 44 & 10 \\
Scopus & 139 & 3 \\
Total & 1.754 & 20
\end{tabular}

Em relação ao delineamento metodológico, os artigos foram categorizados de acordo com a apresentação de seus resultados, tendo sido encontrados maior número de estudos com abordagem estudo de caso $(n=8) \mathrm{e}$ predomínio de pesquisas com abordagem metodológica qualitativa $(n=14)$. Metade dos trabalhos selecionados utilizou mais de um tipo de procedimento de coleta de dados, representado na Tabela 2 pelo termo "outros" $(n=10)$, cujas combinações consistiram em: entrevista e análise documental $(n=2)$; observação participante, entrevista e análise documental $(n=1)$; análise documental de bases governamentais e dados provenientes da literatura ( $n=1)$; observação e entrevistas $(n=3)$; revisão da literatura, análise documental e estudo de caso $(n=1)$; observação, entrevista e atividades com os participantes $(n=1)$; questionário e coleta de amostras de sangue, urina, pressão sanguínea e batimento cardíaco $(n=1)$.

TABELA 2. Metodologia, procedimentos e tipo de pesquisa dos artigos selecionados

\begin{tabular}{ccc}
\hline Metodologia & Número de artigos & Porcentagem (\%) \\
Survey & 5 & 35 \\
Estudo de Caso & 8 & 40 \\
Análise Quase-Experimental & 1 & 5 \\
Outros & 6 & 20 \\
Questionário & Procedimentos & 20 \\
Entrevista & 4 & 30 \\
Outros & 6 & 50 \\
Qualitativa & 10 & 70 \\
Quantitativa & Tipo de pesquisa & 30 \\
\hline
\end{tabular}

No que se refere ao ano de publicação dos artigos selecionados, identificou-se maior predominância de trabalhos nos últimos seis anos (2009-2014). Em relação aos periódicos dos estudos, foram identificados 17 periódicos diferentes, sendo 11 nacionais e 9 de outros países. Os periódicos se inserem em sete áreas principais: administração $(n=5)$, ciências sociais $(n=5)$, saúde $(n=2)$, engenharia de produção $(n=2)$, educação $(n=1)$, trabalho $(n=1)$ e gerenciamento de carreiras $(n=1)$.

$\mathrm{Na}$ Tabela 3 são sistematizados os temas encontrados nas produções científicas das quatro bases eletrônicas de dados analisadas. Verificou-se um predomínio de artigos com foco em terceirização e impactos para a saúde do trabalhador (9 estudos) e em terceirização e impactos nas condições de trabalho (16 estudos). 


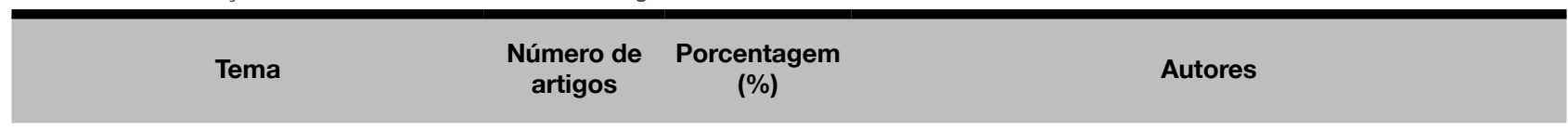

Santos, Lima, Murta e Motta (2009)

Chillida e Cocco (2004)

Costa (2007)

Impactos para a saúde do trabalhador

Impactos para a saúde do trabalhador

9

45

Impactos nas condições de trabalho

16

80
Sugumar, Kumaran, Raj e Xavier (2013)

Hasle (2007)

Seklecka, Marek e Lacala (2013)

Netterstrøm e Hansen (2000)

Zuberi (2011)

Solli-Sæther (2011)

Solli-Sæther (2011)

Azevedo (2014)

Padilha (2014)

Tjandraningsih (2012)

Zuberi e Ptashnick (2011)

Figueiredo, Alvarez, Athayde, Suarez e Pereira (2007)

Petean, Costa e Ribeiro (2014)

Magno e Barbosa (2010)

Nunes (2014)

Zimmermann e Ravishankar (2011)

Rios e Gondim (2010)

Chillida e Cocco (2004)

Costa (2007)

Santos et al. (2009)

Zuberi (2011)

Lopes e Silva (2009)

\section{Impactos para a saúde do trabalhador}

Os estudos encontrados apontaram impactos da terceirização para a saúde do trabalhador. Esses impactos podem ser categorizados em: estresse (Chillida \& Cocco, 2004; Netterstrøm \& Hansen, 2000; Seklecka et al., 2013; Solli-Sæther, 2011; Sugumar et al., 2013; Zuberi, 2011); doenças relacionadas ao trabalho e risco ocupacional (Chillida \& Cocco, 2004; Santos et al., 2009; Zuberi, 2011); sofrimento psíquico (Costa, 2007; Netterstrøm \& Hansen, 2000; Santos et al., 2009; Zuberi, 2011); e falta de suporte à saúde e à segurança (Hasle, 2007).

\section{Estresse}

O estresse foi o impacto mais recorrente nas pesquisas relacionadas aos prejuízos na saúde dos trabalhadores, seja pela forma como é percebido pelos mesmos (Chillida \& Cocco, 2004; Zuberi, 2011), seja mensurado por meio de indicadores físicos (Netterstrøm \& Hansen, 2000; Sugumar et al., 2013). A pesquisa desenvolvida por Sugumar et al. (2013) levantou os sintomas físicos e comportamentos relacionados ao estresse apontados pelos trabalhadores, sendo os principais: problemas gastrointestinais, perda ou ganho de peso, distúrbios do sono, preocupações constantes e irritabilidade.

Seklecka et al. (2013) identificaram a prevalência de fatores associados ao papel profissional, às demandas de trabalho da vida familiar e privada e à falta de oportunidades de crescimento profissional como vinculados ao estresse percebido pelos trabalhadores terceirizados. Já o estudo de Solli-Sæther (2011) identificou dois 
estressores influentes no nível de estresse de funcionários terceirizados: o grau em que o gerente da empresa que contrata os serviços espera que os trabalhadores terceirizados ajam como subordinados, e a expectativa de que esses trabalhadores adotem comportamentos de flexibilidade, troca de informações e solidariedade. A partir do estudo, pode-se verificar que a natureza dos estressores está diretamente relacionada às questões organizacionais, entre elas o papel exercido pela liderança direta e as expectativas da organização em relação aos profissionais.

\section{Doenças relacionadas ao trabalho e risco ocupacional}

$\mathrm{O}$ desenvolvimento de doenças relacionadas diretamente à natureza das atividades e o risco de acidentes também é percebido por estes trabalhadores (Santos et al., 2009; Zuberi, 2011), principalmente em ocupações que exigem intenso trabalho físico. Na pesquisa realizada por Chillida e Cocco (2004), alguns dos trabalhadores terceirizados atuantes em um serviço de limpeza hospitalar relataram sofrer de doenças como tendinite e dermatite, e muitos associaram o risco ocupacional à utilização de produtos de limpeza, ao trabalho repetitivo e à probabilidade de se envolverem em acidentes com instrumentos perfurocortantes e fluídos biológicos.

Além disso, o risco de acidentes também pode ser intensificado pelo fato de que muitos terceirizados trabalham em mais de um serviço como meio de conseguir custear todas as despesas, uma vez que o salário dos trabalhadores terceirizados geralmente é mais baixo que a média dos trabalhadores fixos (Zuberi, 2011).

\section{Sofrimento psíquico}

Sabe-se que o controle do trabalhador sobre suas atividades é algo essencial para a construção da saúde, de modo que o trabalho se torna interessante para o indivíduo quando há possibilidade de expressão e criatividade e, quando este aspecto é ameaçado, pode ter início um processo de adoecimento psíquico, o que muitas vezes é negligenciado pelas empresas e pelo próprio sujeito (Santos et al., 2009). Nesse sentido, o estudo realizado por esses pesquisadores, cujos participantes eram garis terceirizados, indicou que os elementos da dinâmica do ambiente de trabalho que se destacaram como prejudiciais à saúde foram o esvaziamento do sentido do trabalho, a anulação da identidade profissional e as relações baseadas em constrangimento, desrespeito e autoritarismo.

Os dados da pesquisa de Netterstrøm e Hansen (2000) com motoristas corroboram os achados anteriores. Os trabalhadores terceirizados relataram pouco significado atribuído ao trabalho, baixa previsibilidade, baixo suporte entre os colegas e pouca satisfação no trabalho. Esses fatores geram uma importante carga emocional para os trabalhadores e podem desencadear o início de um processo de adoecimento psíquico, assim como fracos vínculos com a empregadora, sentimento de inferioridade, falta de acompanhamento e oportunidades de crescimento profissional (Costa, 2007) e sentimento de insegurança (Zuberi, 2011).

\section{Impactos nas condições de trabalho}

Dos artigos selecionados, 16 abordam a influência que a terceirização exerce sobre as condições de trabalho dos trabalhadores terceirizados, sendo que esses impactos foram categorizados da seguinte maneira: vínculo, identidade profissional e identificação com a empresa (Costa, 2007; Lopes \& Silva, 2009; Magno \& Barbosa, 2010; Rios \& Gondim, 2010; Solli-Sæther, 2011); precarização das condições de trabalho (Azevedo, 2014; Costa, 2007; Magno \& Barbosa, 2010; Petean et al., 2014; Zuberi \& Ptashnick, 2011); diferenças de benefícios e tratamento (Figueiredo et al., 2007; Magno \& Barbosa, 2010; Padilha, 2014; Petean et al., 2014; Rios \& Gondim, 2010; Tjandraningsih, 2012; Zimmermann \& Ravishankar, 2011); e diminuição dos direitos trabalhistas e salário (Chillida \& Cocco, 2004; Costa, 2007; Figueiredo et al., 2007; Santos et al., 2009; Tjandraningsih, 2012; Zuberi, 2011).

\section{Vínculo, identidade profissional e identificação com a empresa}

O estudo realizado por Solli-Sæther (2011) examinou a influência das expectativas de funcionários fixos e as influências da ambiguidade de papéis na performance do trabalho, no compromisso afetivo e na intenção de sair da organização em trabalhadores que passaram pelo processo de terceirização. Os resultados apontaram altos índices de ambiguidade de papéis percebida, relacionados à diminuição da performance nas tarefas.

Os dados também indicaram que os trabalhadores terceirizados não se sentem pertencentes à empresa, que os gerentes da empresa contratante esperam que eles ainda ajam como subordinados, e que a exigência de comportamentos de flexibilidade, troca de informações e solidariedade no ambiente de trabalho afetam negativamente a percepção de ambiguidade de papéis (Solli-Sæther, 2011). 
Analogamente a esse estudo, Lopes e Silva (2009) realizaram uma pesquisa que visou compreender como os profissionais terceirizados de Tecnologia da Informação (TI) percebem suas chances de carreira e suas relações com as organizações, analisando possíveis implicações dessas percepções para os indivíduos e seus empregadores. Constatou-se que, frente à instabilidade em suas relações de trabalho, os trabalhadores terceirizados priorizam seus interesses pessoais e de carreira, reforçando sua identidade como "profissionais de TI" e não como integrantes de uma organização, destacando a empregabilidade como responsabilidade do indivíduo, investimento individual no desenvolvimento de habilidades, e carreiras sem fronteiras frente à perspectiva transacional que as relações de trabalho estão, cada vez mais, assumindo (Lopes \& Silva, 2009).

Costa (2007) realizou um estudo com 20 trabalhadores terceirizados que atuavam na Procuradoria-Geral de Justiça do Estado do Rio Grande do Sul em serviços de apoio, como limpeza, recepção e telefonia. Os participantes relataram dificuldade em estabelecer relacionamentos de longo prazo, em criar laços de lealdade e em desenvolver sentimentos de segurança e de identidade pessoal vinculada à carreira devido à interação tanto com a empresa para a qual prestam serviços quanto com a empregadora, além do aspecto temporário que o trabalho terceirizado assume. A empregadora frequentemente limita-se a aspectos pontuais e transicionais de seus funcionários, o que dificulta o estabelecimento de vínculos e a identificação com essa empresa por parte dos terceirizados.

De acordo com os resultados do levantamento realizado por Rios e Gondim (2010), destacam-se diferentes padrões de contratos psicológicos entre trabalhadores terceirizados e não terceirizados. Essas diferenças são mais expressivas em relação ao investimento numa relação de trabalho duradoura, na expectativa de equidade e na transparência em relação a anseios e problemas da parte dos não terceirizados. Além disso, os dados obtidos pelos autores apontam que o profissional terceirizado é caracterizado como um sujeito que possui um vínculo mais frágil de trabalho, aspecto que se reflete nas expectativas que ele possui em relação às suas obrigações e às do empregador, por exemplo.

Magno e Barbosa (2010) destacam a existência de identidades sociais distintas entre os trabalhadores diretos e terceirizados. Segundo os autores, a figuração identitária entre os profissionais terceirizados e os efetivos fundamenta-se na rede de proteção social que a empresa disponibiliza aos seus empregados e que as prestadoras de serviços não oferecem. Isso propicia uma sensação de segurança social significativamente menor por parte dos terceirizados em relação aos trabalhadores diretos.

\section{Precarização das condições de trabalho}

Os estudos de Petean et al. (2014) e Zuberi e Ptashnick (2011) com funcionários terceirizados de limpeza em hospitais enfatizam aspectos que sinalizam tal precarização decorrente da terceirização. Evidenciou-se a adoção desta prática como um dos fatores desencadeadores de dificuldades encontradas por profissionais terceirizados em seu ambiente de trabalho. Os trabalhadores relataram que a terceirização estava associada à deterioração das condições de trabalho, posto que a terceirização afetava negativamente as experiências e a qualidade de trabalho em seu ambiente laboral. Foram relatados pelos trabalhadores terceirizados trabalho em equipes reduzidas, acidentes de trabalho, alta rotatividade, excesso de trabalho e falta de treinamento. Relatos sobre a falta de treinamento e acompanhamento também foram observados em outros estudos (Costa, 2007; Magno \& Barbosa, 2010), contribuindo para a percepção de sentimentos de inferioridade e inadequação relacionados à execução de atividades como profissional terceirizado (Costa, 2007).

Já na pesquisa de Azevedo (2014), verificou-se a terceirização profissional de consultorias empresariais de serviço social no Estado de São Paulo, analisando o processo de precarização das condições de trabalho do assistente social. A autora reforça os resultados apresentados nos estudos anteriores, destacando que a precarização das organizações e das condições de trabalho se expressa na intensificação do trabalho, na restrição da autonomia, nas metas, pressões, extensão da jornada, polivalência e rotatividade, entre outros.

\section{Diferenças de benefícios e tratamento}

Estudos apontam diferenças na qualificação, treinamento, remuneração e benefícios entre o trabalhador terceirizado e o efetivo (Figueiredo et al., 2007; Magno \& Barbosa, 2010; Rios \& Gondim, 2010). De acordo com Figueiredo et al. (2007), cujo estudo desenvolveu-se no setor petrolífero, o número menor de dias de folga em terra para os terceirizados, deixando-os mais vulneráveis aos riscos do trabalho em turnos na plataforma, 
é um dos aspectos de precarização das condições de trabalho dos trabalhadores terceirizados. Além disso, o estudo apontou inúmeros casos de subnotificações de acidentes graves com terceiros.

Ademais, os estudos destacam que a precarização contribui para a fragmentação dos coletivos de trabalho. As diferentes práticas de relações de trabalho permanentes e contratuais ocasionam a fragmentação dos trabalhadores, de acordo com suas respectivas posições de trabalho, visto que essa diferenciação causa a estratificação e a distância social na força de trabalho da fábrica, afetando a solidariedade e a consciência comum dos funcionários como um grupo de trabalhadores (Figueiredo et al., 2007; Tjandraningsih, 2012).

Os trabalhadores terceirizados tendem a manifestar sentimento de desvalorização em relação aos outros profissionais, exprimindo os reflexos da terceirização em suas vidas na constatação de um menor valor humano e social (Petean et al., 2014). O estudo de Nunes (2014) corrobora esses aspectos. Ele constatou que a contratação terceirizada, no caso de serventes de limpeza, afeta negativamente a dinâmica do reconhecimento, uma vez que há discriminação pelo fato de serem terceirizados e não efetivos.

De modo semelhante, de acordo com Padilha (2014), os trabalhadores de limpeza terceirizados relataram ter percebido desigualdade e humilhação no ambiente de trabalho, principalmente devido à relação hierárquica que os coloca em posição inferior em relação aos outros trabalhadores.

O estudo realizado por Zimmermann e Ravishankar (2011) também corrobora esses aspectos. Com o objetivo de examinar como o trabalho em um arranjo organizacional envolvendo terceirização tem influenciado a reconfiguração dos papéis de identidade profissional, a pesquisa constatou que os trabalhadores terceirizados vivenciam frustrações por receberem tarefas rotineiras e que proporcionam pouco crescimento profissional, e também um status inferior percebido na relação entre a empresa contratante e a empresa que fornece serviços terceirizados.

\section{Diminuição dos direitos trabalhistas e salário}

As pesquisas de Figueiredo et al. (2007) e de Tjandraningsih (2012) destacam a perda de direitos trabalhistas por parte dos profissionais terceirizados, uma vez que algumas empresas terceirizadas atuam de forma ilegal, infringindo uma série de itens da legislação trabalhista nos contratos de trabalhadores terceirizados. Essa nova modalidade de relações de trabalho enfraquece o papel do Estado na gestão das relações laborais e na proteção dos trabalhadores, insere o processo de informalização no setor formal, reduz a qualidade de vida e estimula a corrupção e a exploração (Tjandraningsih, 2012).

Sabe-se que o salário dos trabalhadores terceirizados é, em média, mais baixo que o salário dos efetivos e que, devido a isso, muitos terceirizados acabam trabalhando em mais de um emprego para conseguir complementar a renda (Chillida \& Cocco, 2004; Costa, 2007; Santos et al., 2009; Zuberi, 2011). De acordo com pesquisa realizada por Zuberi (2011), mais de 70\% dos trabalhadores terceirizados entrevistados relataram alguma dificuldade para lidar com os gastos mensais, e 30\% indicaram que trabalhavam em dois ou mais locais para pagar as contas, sendo que alguns também contavam com empréstimos e com a ajuda de familiares para isso.

\section{DISCUSSÃO}

Os resultados permitiram o acesso aos estudos recentes realizados em relação à temática da terceirização e seus impactos para os trabalhadores. Foi possível identificar que a terceirização impacta a saúde e as condições de trabalho dos funcionários submetidos a tal prática. Constatou-se que as condições de trabalho a que são submetidos os trabalhadores terceirizados, como diferenças salariais, de benefícios e de tratamento (Figueiredo et al., 2007; Magno \& Barbosa, 2010; Padilha, 2014; Petean et al., 2014; Rios \& Gondim, 2010; Tjandraningsih, 2012; Zimmermann \& Ravishankar, 2011), impactam a saúde dos profissionais e podem gerar sobrecarga física e psíquica (Costa, 2007; Santos et al., 2009), além de desencadear o aparecimento de doenças relacionadas ao trabalho (Chillida \& Cocco, 2004; Santos et al., 2009; Zuberi, 2011). Os estudos sobre doenças relacionadas ao trabalho de terceirizados apontam a existência de doenças físicas, mas principalmente um aumento de incidência de doenças mentais relacionadas ao trabalho, como o estresse (Chillida \& Cocco, 2004; Netterstrøm \& Hansen, 2000; Seklecka et al., 2013; Sugumar et al., 2013; Zuberi, 2011).

Também foram encontrados estudos que relacionavam aspectos organizacionais, como a falta de treinamento, a maiores índices de acidentes e risco ocupacional (Santos et al., 2009; Zuberi, 2011). Os estudos apontam ainda uma diminuição na performance das tarefas, por parte dos trabalhadores terceirizados, em virtude de 
altos índices de ambiguidade de papéis (Solli-Sæther, 2011) e diminuição da identificação do trabalhador com a empresa (Costa, 2007), além de alteração no vínculo com o trabalho (Rios \& Gondim, 2010), que resulta em taxas mais altas de rotatividade (Azevedo, 2014; Petean et al., 2014; Zuberi \& Ptashnick, 2011), absenteísmo e comprometimento com o trabalho (Santos et al., 2009).

Verificou-se, ainda, uma predominância de estudos do tipo estudo de caso, com abordagem qualitativa. Foram encontrados poucos estudos do tipo levantamento, com abordagens teórico-metodológicas mais robustas, para verificar relações causais entre o processo de terceirização e o estresse, doenças relacionadas ao trabalho, riscos de acidentes e sofrimento psíquico.

Sendo assim, pode-se concluir que a terceirização parece ter impactos negativos sobre a saúde e qualidade de vida do trabalhador, ocasionando doenças e sofrimento relacionados ao trabalho. No entanto, os fatores que podem resultar na deterioração da saúde dos profissionais terceirizados foram estudados predominantemente de forma isolada nos artigos, não sendo possível identificar as inter-relações entre os mesmos. Assim, parece existir uma lacuna de estudo das inter-relações entre estes fatores, de forma a investigar o processo saúdedoença desses profissionais.

Dessa forma, a partir da análise da literatura, verificou-se a necessidade da realização de estudos que identifiquem as inter-relações entre as condições de trabalho a que são submetidos os trabalhadores terceirizados e os processos de saúde-adoecimento. A partir do estudo dessas inter-relações, espera-se que se torne possível sugerir mudanças a serem implementadas por empresas terceiras que resultem em melhorias nas condições de trabalho a que são submetidos esses profissionais.

\section{REFERÊNCIAS}

Azevedo, F. C. (2014). Consultoria empresarial de serviço social: Expressões da precarização e da terceirização profissional. Serviço Social \& Sociedade, 118, 318-338. doi: 10.1590/S0101-66282014000200006.

Brooks, N. (2006). Understanding IT outsourcing and its potential effects on IT workers and their environment. The Journal of Computer Information Systems, 46(4), 46-53.

Chillida, M. S. P., \& Cocco, M. I. M. (2004). Saúde do trabalhador e terceirização: Perfil de trabalhadores de serviço de limpeza hospitalar. Revista Latino-Americana de Enfermagem, 12(2), 271-276. doi: 10.1590/S0104-11692004000200018.

Costa, S. G. (2007). O pai que não é o patrão: Vivências de sujeitos terceirizados no Ministério Público do estado do Rio Grande do Sul. Organizações \& Sociedade, 14(42), 97-113. doi: 10.1590/S1984-92302007000300006.

Davis-Blake, A., \& Broschak, J. P. (2009). Outsourcing and the changing nature of work. Annual Review of Sociology, 35, 321340. doi: 10.1146/annurev.soc.34.040507.134641.

Figueiredo, M., Alvarez, D., Athayde, M., Suarez, J. D., \& Pereira, R. (2007). Reestruturação produtiva, terceirização e relações de trabalho na indústria petrolífera offshore da Bacia de Campos (RJ). Gestão \& Produção, 14(1), 55-68. doi: 10.1590/ S0104-530X2007000100006.

Hasle, P. (2007). Outsourcing and employer responsibility: A case study of occupational health and safety in the Danish public transport sector. Relations Industrielles, 62(1), 96-117. doi: 10.7202/015799ar.

Hernandes, E., Zamboni, A., Fabbri, S., \& Thommazo, A. (2012). Using GQM and TAM to evaluate StArt - A tool that supports systematic review. CLEI Eletronic Journal, 15(1), 3-3.

Lima, J. C. (2010). A terceirização e os trabalhadores: Revisitando algumas questões. Cadernos de Psicologia Social do Trabalho, 13(1), 17-26. doi: http://dx.doi.org/10.11606/issn.1981-0490.v13i1p17-26

Lopes, A. L. S. V., \& Silva, J. R. G. (2009). Expectativas profissionais no discurso de terceirizados em TI. Revista de Administração de Empresas - Eletrônica, 8(2). doi: 10.1590/S1676-56482009000200002.

Magno, A., \& Barbosa, S. (2010). A naturalização da identidade social precarizada na indústria do alumínio primário paraense. Sociologias, 12(23), 268-303. doi: 10.1590/S1517-45222010000100010.

Netterstrøm, B., \& Hansen, A. M. (2000). Outsourcing and stress: Physiological effects on bus drivers. Stress Medicine, 16(3), 149-160. doi: 10.1002/(SICI)1099-1700(200004)16:3<149::AID-SMI845>3.0.CO;2-X.

Nunes, J. H. (2014). Dilemas identitários no mundo dos serviços: Da invisibilidade à interação. Sociologias, 16(35), 238-273. doi: 10.1590/S1517-45222014000100009.

Padilha, V. (2014). Nojo, humilhação e controle na limpeza de shopping centers no Brasil e no Canadá. Caderno CRH, 27(71), 329-346. doi: 10.1590/S0103-49792014000200008. 
Petean, E., Costa, A. L. R. C., \& Ribeiro, R. L. R. (2014). Repercussões da ambiência hospitalar na perspectiva dos trabalhadores de limpeza. Trabalho, Educação e Saúde, 12(3), 615-635. doi: 10.1590/1981-7746-sip00005.

Petticrew, M., \& Roberts, H. (2006). Systematic reviews in the social sciences: A practical guide. Recuperado de http:// onlinelibrary.wiley.com/book/10.1002/9780470754887

Rios, M. C., \& Gondim, S. M. G. (2010). Contratos psicológicos e terceirização: Um estudo das relações entre vínculos e as práticas de gestão de pessoas. Organizações \& Sociedade, 17(55), 689-703. doi: 10.1590/S1984-92302010000400006.

Santos, M. C. O., Lima, F. P. A., Murta, E. P., \& Motta, G. M. V. (2009). Desregulamentação do trabalho e desregulamentação da atividade: O caso da terceirização da limpeza urbana e o trabalho dos garis. Produção, 19(1), 202-213. doi: 10.1590/S010365132009000100013.

Seklecka, L., Marek, T., \& Lacala, Z. (2013). Work satisfaction, causes, and sources of job stress and specific ways of coping: a case study of white-collar outsourcing service employees. Human Factors and Ergonomics in Manufacturing \& Service Industries, 23(6), 590-600. doi: 10.1002/hfm.20554.

Solli-Sæther, H. (2011). Transplants' role stress and work outcome in IT outsourcing relationships. Industrial Management \& Data Systems, 111(2), 227-245. doi: 10.1108/02635571111115155.

Sugumar, D., Kumaran, C. K. M., Raj, P. J., \& Xavier, S. J. (2013). Addressing health related challenges faced by the business process outsourcing (BPO) employees by stress. African Journal of Business Management, 7(12), 906-914. doi: 10.5897/ AJBM2013.1599

Tjandraningsih, I. (2012). State-sponsored precarious work in Indonesia. American Behavioral Scientist, 57(4), 403-419. doi: $10.1177 / 0002764212466236$.

Zimmermann, A., \& Ravishankar, M. N. (2011). Collaborative IT offshoring relationships and professional role identities: Reflections from a field study. Journal of Vocational Behavior, 78(3), 351-360. doi: 10.1016/j.jvb.2011.03.016.

Zoltowski, A. P. C., Costa, A. B., Teixeira, M. A. P., \& Koller, S. H. (2014). Qualidade metodológica das revisões sistemáticas em periódicos de psicologia brasileiros. Psicologia: Teoria e Pesquisa, 30(1), 97-104. doi: 10.1590/S0102-37722014000100012.

Zuberi, D. (2011). Contracting out hospital support jobs: The effects of poverty wages, excessive workload, and job insecurity on work and family life. American Behavioral Scientist, 55(7), 920-940. doi: 10.1177/0002764211407835.

Zuberi, D. M., \& Ptashnick, M. B. (2011). The deleterious consequences of privatization and outsourcing for hospital support work: The experiences of contracted-out hospital cleaners and dietary aids in Vancouver, Canada. Social Science \& Medicine, 72(6), 907-911. doi: 10.1016/j.socscimed.2010.12.024. 